\title{
Superfluid-Mott transitions and vortices in the Jaynes-Cummings-Hubbard lattices with time-reversal-symmetry breaking
}

\author{
A. L. C. Hayward and A. M. Martin \\ School of Physics, University of Melbourne, Victoria 3010, Australia \\ (Received 20 October 2015; revised manuscript received 8 December 2015; published 22 February 2016)
}

\begin{abstract}
We investigate the ground-state behavior of Jaynes-Cummings-Hubbard lattices in the presence of a synthetic magnetic field, via a Gutzwiller ansatz. Specifically, we study the superfluid-Mott transition and the formation of vortex lattices in the superfluid regime. We find a suppression of the superfluid fraction due to the frustration induced by the incommensurate magnetic and spacial lattice lengths. We also predict the formation of triangular vortex lattices inside the superfluid regime.
\end{abstract}

DOI: 10.1103/PhysRevA.93.023828

\section{INTRODUCTION}

A Jaynes-Cummings-Hubbard (JCH) lattice [1-3] consists of an array of coupled photonic cavities, with each cavity mode coupled to a two-level atom [Figs. 1(a) and 1(b)]. The $\mathrm{JCH}$ model is predicted to exhibit a number of solid state phenomena, including Mott and superfluid phases [2], supersolid phases [4], semiconductor physics [5], the Josephson effect [6], metamaterial properties [7], Bose-glass phases [8], and, in the presence of synthetic magnetic fields, fractional quantum Hall states [9].

In this paper we examine how the introduction of a synthetic magnetic field affects the superfluid-Mott insulator phase transition and leads to the formation of vortices in the superfluid phase. In Sec. II we introduce the formalism and numerical techniques under which we will study the JCH model. Section III examines the properties of the phase transition and how the presence of a synthetic magnetic field affects the superfluid-Mott transition. Our results suggest that frustration due to incommensurate magnetic or spacial lattices suppresses the emergence of a superfluid phase at strong nonlinear interaction strengths. Finally, in Sec. IV we study the formation of vortices in the photonic field in the superfluid regime. We find that vortices in the photonic field of the JCH behave in a similar way to those found in continuous superfluids, including the formation of a triangular Abrikosov lattice.

\section{JAYNES-CUMMINGS-HUBBARD MODEL}

Each cavity [Fig. 1(b)] in the JCH lattice is described by the Jaynes-Cummings (JC) Hamiltonian ( $\hbar=1)$

$$
H^{\mathrm{JC}}=\omega L+\Delta \sigma^{+} \sigma^{-}+\beta\left(\sigma^{+} a+\sigma^{-} a^{\dagger}\right),
$$

where $a$ is the photonic annihilation operator, $\sigma^{ \pm}$are the atom raising and lowering operators, $\Delta$ is the atom-photon detuning, and $\beta$ is the coupling energy. For $n$ photons the state $|g(e), n\rangle$ forms the single cavity basis, where $g(e)$ denote the ground (excited) state of the atom. Throughout this paper we shall be using the dressed basis

$$
| \pm, \ell\rangle=\frac{\beta \sqrt{\ell}|g, \ell\rangle+[-\Delta / 2 \pm \chi]|e, \ell-1\rangle}{\sqrt{2 \xi^{2} \mp \xi \Delta}},
$$

with

$$
\chi=\sqrt{\ell \beta^{2}+\Delta^{2} / 4}
$$

the generalized Rabi frequency, and $\ell$ refers to the total number of excitations in the cavity. In this basis the energy of a single cavity, with $\ell$ excitations is

$$
E_{ \pm, \ell}=\ell \omega \pm \chi-\Delta / 2 .
$$

Since $H^{\mathrm{JC}}$ commutes with the total excitation number operator, $L=a^{\dagger} a+\sigma^{+} \sigma^{-}$the total excitations in the cavity, $\ell$, is a good quantum number. The eigenstates of Eq. (1) are termed polaritons, superpositions of atomic and photonic excitations, and are a function of $\ell$ and $\Delta / \beta$.

The ground-state energy of JC cavity depends nonlinearly on the number of excitations. That is, the atom-cavity interaction induces an effective particle-particle interaction that drives the nontrivial physics seen in JC systems. Figure 2 shows how this energy (per excitation) scales with the excitation number $(\ell)$. Furthermore, the effective interaction is repulsive, as we can see from inspection of the figure [or directly in Eq. (4)], whereby the energy per excitation is increasing as the total number is increased.

Figure 2 also shows how the effective nonlinearity changes with the cavity detuning. In the limit where the atomic energy is far below that of the photon $(\Delta / \beta \ll 0)$, the interaction becomes stronger, as the addition of further excitations after the first must sit mostly in the cavity. In the opposite limit, excitations are almost completely photonic, and the presence of the cavity atom can be thought of as mediating a photon-photon interaction, $U_{2}^{\text {eff }}$, which is approximately $U_{2}^{\text {eff }}=\beta^{4} / \Delta^{3}$. Thus the atomic energy offers a knob by which to tune the interaction strengths in JC systems.

The JCH model describes a tight-binding JC lattice [Fig. 1(a) with $\alpha=0$ ]:

$$
H^{\mathrm{JCH}}=H^{\mathrm{JC}}+K=\sum_{i}^{N} H_{i}^{\mathrm{JC}}-\sum_{\langle i, j\rangle} \kappa_{i j} a_{i}^{\dagger} a_{j},
$$

where $\kappa_{i j}$ is the photon tunneling rate between cavities $i$ and $j$ and the sum over $\langle i, j\rangle$ is between nearest neighbors only.

Exact solutions to $H^{\mathrm{JCH}}$ are known in two limits: $\kappa / \beta \rightarrow 0$, and $\beta / \kappa \rightarrow 0$. In the former limit, each cavity is decoupled, and the eigenstates are simply product states of individual cavities, described by the solutions in Eq. (2). In the opposite limit, where $\beta / \kappa \rightarrow 0$, the photonic and atomic degrees of freedom decouple. This removes the nonlinearity from the photonic field, and any interatomic coupling, 
(a)

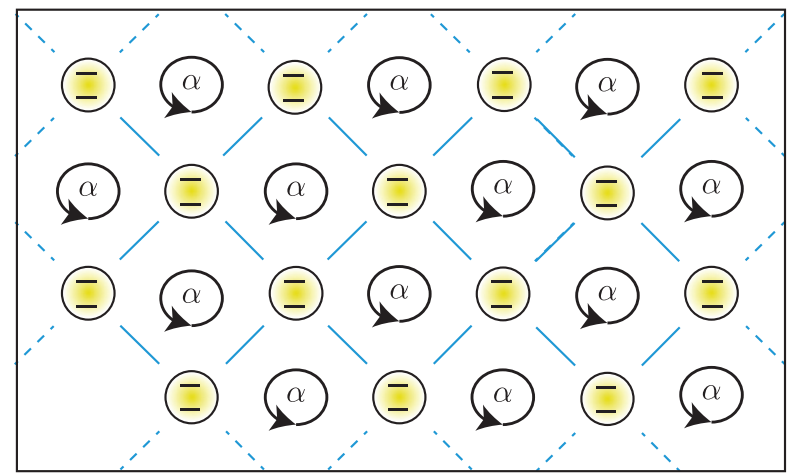

(b)

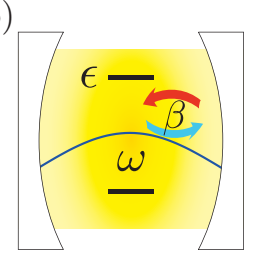

(c)

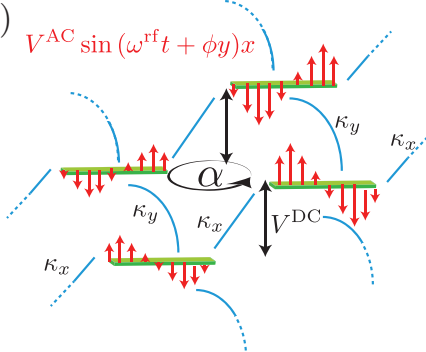

FIG. 1. (a) Schematic of a square JCH lattice with a constant synthetic magnetic field. Photons moving around a plaquette acquire a phase $2 \pi \alpha$ (b) A single-mode photonic cavity with frequency $\omega$ coupled to a two-level atom with strength $\beta$. (c) Scheme for breaking time reversal symmetry in photonic cavities: a potential $V=\left[V^{\mathrm{DC}}+V^{\mathrm{AC}} \sin \left(\omega^{\mathrm{rf}}+2 \pi \alpha y\right)\right] x$, where $x$ and $y$ are in units of the lattice spacing, is applied to the cavities by dynamically tuning $\omega$. The phase offset, $2 \pi \alpha$, along $y$ results in the synthetic magnetic field seen in (a).

again reducing the eigenstates to product states of single particle.

\section{A. The Gutzwiller ansatz}

For large system sizes, the exact quantum mechanical problem of the JCH model is too computationally difficult to approach directly, either analytically or numerically. Various approximations have been used to study the superfluid Mott insulator phase transition in the JCH model: mean-field approximations [2,10], Monte-Carlo simulations [11], and a density matrix renormalization group $[12,13]$. In general, the broad structure found through the mean-field approach is confirmed by the more rigorous methods.

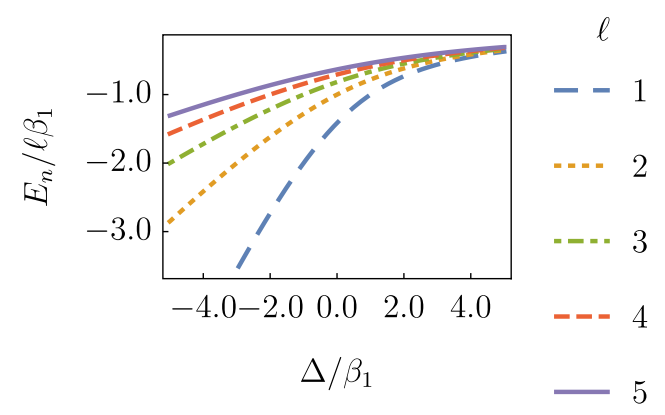

FIG. 2. Average energy per excitation in a Jaynes-Cummings cavity, as a function of $\Delta$ for $\ell=1,5$.

In this paper we use a Gutzwiller ansatz to study the JCH ground-state wave function. The Gutzwiller ansatz imposes a trial wave function that is a product state of individual lattice sites:

$$
P_{\ell}\left|\Psi^{\mathrm{GW}}\right\rangle=\prod_{i}^{\text {sites }}\left|\psi_{i}\right\rangle,
$$

where $P_{\ell}$ projects onto an excitation number eigenstate. Applying $\left|\Psi^{\mathrm{GW}}\right\rangle$ to the JCH Hamiltonian yields an effective Hamiltonian that is a sum over single cavity Hamiltonians:

$$
H_{\mathrm{eff}}^{\mathrm{JCH}}=\sum_{i}^{N} H_{i}^{\mathrm{JC}}-\sum_{\langle i, j\rangle}\left[\kappa_{i j} \psi_{j}^{\star}\left(a_{i}-\frac{1}{2} \psi_{i}\right)+\text { H.c. }\right],
$$

where we have introduced the notation $\psi_{i}=\left\langle\psi_{i}\left|a_{i}\right| \psi_{i}\right\rangle$. With this effective Hamiltonian in hand, the Gutzwiller ground state can be found by minimizing the energy of $H_{\mathrm{eff}}^{\mathrm{JCH}}$.

The local superfluid order parameter $\vec{\psi}=\left\{\psi_{i}\right\}$ determines the nature of the ground state. From Eq. (7) it follows that the off-diagonal elements of the single-particle density matrix are determined by $\vec{\psi}: \rho_{i j}^{1}=\psi_{i} \psi_{j}^{\star}$. The system can be said to be superfluid whenever there are system-wide connected nonzero $\psi_{i}$. The Mott insulating phase is characterized by $\psi_{i}=0$ across the whole system.

The effective Hamiltonian arrived at though the Gutzwiller procedure removes the restriction of the system to a specific number of excitations, since, except in the $\vec{\psi}=0$ case, the Hamiltonian does not conserve the particle number. However, it is possible to restrict the particle density implicitly though the introduction of a chemical potential:

$$
H=H^{\mathrm{JCH}}-\mu L .
$$

That is, the system is now studied in the grand-canonical ensemble, and the system's ground state at the desired density is found by varying the chemical potential. In the case of the effective Hamiltonian in Eq. (7), this is necessary since this Hamiltonian does not preserve photon number.

There has been some confusion in the literature $[2,14]$ as to the physical significance, or meaning, of the chemical potential in photonic systems. From a mathematical perspective, the chemical potential introduced in this manner acts simply as a Lagrange multiplier. The choice of $\mu$ defines an excitation density for the ground state. The chemical potential can also appear as a real physical quantity. Consider the JCH weakly coupled to a bath of photons, all with frequency $\omega_{b}$ such that $\omega-\omega_{b}=\mu$. Assuming a mechanism for thermalization, such a coupling of the bath to atomic or photonic modes, the pump will act to drive the system to some ground state, determined by $\mu=\omega_{\text {pump. }}$. Weakly blue band pumping will act to create a positive chemical potential for photons in the system. Although a JCH system under such pumping, when paired with photon losses, will exhibit nonequilibrium dynamics, when these effects are small, the system will be well described by Eq. (8) in the ground state [15].

For real world cavity lattices, the photons in the system will not be conserved, due to dissipation. In contrast to cold atom systems, where some set number of particles can be loaded into the lattice, a photonic lattice is driven by a laser and will be in a steady state where losses and driving equilibrate [16]. Still, for 
an array of high-quality cavities, one may consider a regime of weak pumping, and weak losses, such that the mean-field approximation made here captures the broad physics of the system [17].

\section{B. Synthetic magnetic field}

An artificial magnetic field may be realized via the introduction of some time reversal symmetry breaking interaction. A number of techniques have been proposed to achieve this [8,18-22]. For example, one may exploit a time-dependent potential to induce magnetic flux across the lattice [as in Fig 1(c)] [21], thereby explicitly breaking the time symmetry of the system. A similar strategy is proposed in Ref. [8], where the authors utilize a time-dependent intersite coupling to induce a synthetic magnetic field. Alternatively, effective coupling to real magnetic fields can be used, such as in the proposal by Koch et al. [20], or other means, such as the use of optically polarized media [18] or via atomically mediated intersite coupling [19].

Several of these methods involve extra degrees of freedom or the introduction of time-dependent terms into the Hamiltonian. However, via an appropriate gauge transformation or adiabatic elimination, all of the techniques reduce to a system in which the intersite coupling has acquired an uneliminateable complex phase factor, equivalent to a magnetic field.

Defining the magnetic field by a vector potential $\mathbf{A}(x)$ results in minimal substitution, $\mathbf{p} \rightarrow \mathbf{p}-q \mathbf{A}(x)$. On a tightbinding lattice, a vector potential $\mathbf{A}$ manifests as a complex hopping rate $\kappa_{i j} \rightarrow \kappa_{i j} e^{i 2 \pi \theta_{i j}}$, where $2 \pi \theta_{i j}=\int_{r_{i}}^{r_{j}} \mathbf{A}(r) d r$. Gauge symmetry implies that the only physically important parameter is the total phase, $2 \pi \alpha$, picked up around a closed loop, where $\alpha=\Phi / \Phi_{0}$ is the fraction of flux quanta through the loop. A uniform magnetic field through the two-dimensional lattice corresponds to a constant $\alpha$ for all plaquettes on the lattice. Factors of $2 \pi$ in the phase around a loop are physically inconsequential, so we only need consider $\alpha \in[0,1)$.

\section{PHASE DIAGRAM}

The simplest situation in which the superfluid-Mott transition occurs is in the homogeneous lattice, with $\alpha=0$. Since the mean-field ground state of our Hamiltonian is invariant under translations along lattice vectors it is possible to significantly simplify the problem to that of a single site. That is, since $\left|\psi_{i}\right\rangle=\left|\psi_{j}\right\rangle \forall i, j$ we have just a single $\psi=\left\langle a_{i}\right\rangle$ that characterizes the whole ground state. Hence for $\alpha=0$ we find the well-known result [1-3] [Fig. 3(a)] that the parameter space is separated into two distinct phases. For low hopping strength $\bar{\kappa}=\kappa / \beta$, we find lobes of vanishing superfluid order parameter, i.e., Mott-insulating phases as shown in Fig. 3(a). Each lobe corresponds to a state with an integer number of strongly localised excitations per site. For example the first (second) Mott lobe, with $\bar{\mu}=(\mu-\omega) / \beta<-1[-1<$ $\bar{\mu}=(\mu-\omega) / \beta<-0.4]$ corresponds to the states $|-, 0\rangle$ $(|-, 1\rangle)$. For low chemical potential, there are no excitations in the system. Raising the chemical potential, the block is successively filled with one, two, and more excitations per site. At sufficiently large $\bar{\kappa}$, the system undergoes a phase

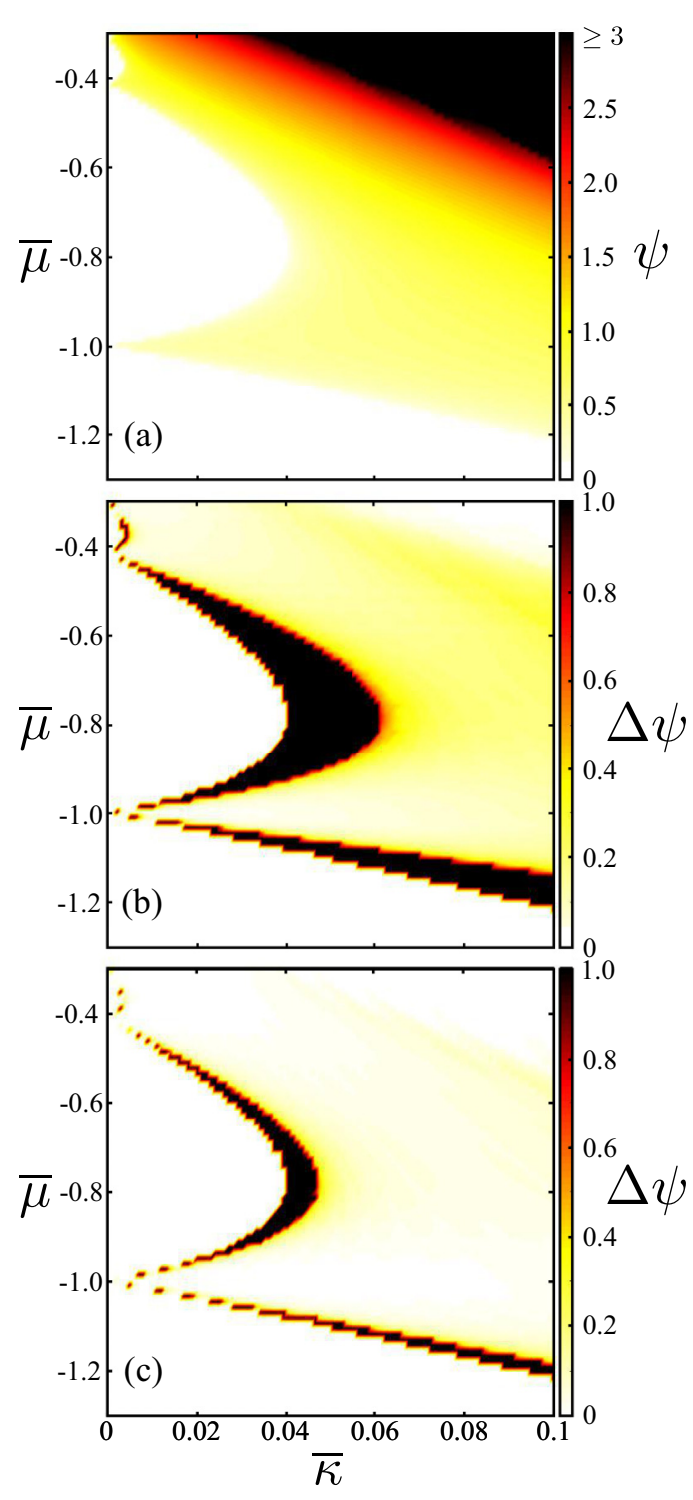

FIG. 3. (a) The superfluid order parameter $(\psi)$ as a function of the chemical potential $\bar{\mu}$ and the interactive hopping $\bar{\kappa}$, with a lattice of $4 \times 5$ sites in the absence of synthetic magnetic field $(\alpha=0)$. (b, c) $\Delta \psi$ as a function of the chemical potential $\bar{\mu}$ and the interactive hopping $\bar{\kappa}$, for $|\alpha|=2 / 5$ and $3 / 5$ : (b) and $|\alpha|=1 / 10$ and $9 / 10$; (c), with $\epsilon=0.001$. For (a)-(c) we have introduced the following dimensionless parametrization: $\bar{\kappa}=\kappa / \beta, \bar{\mu}=(\mu-\omega) / \beta$, and $\bar{\Delta}=$ $\Delta / \beta=0$.

transition into a phase of finite superfluid order parameter [Fig. 3(a)]. The excitations are homogeneously distributed and delocalized, i.e., the system is in a superfluid state.

The superfluid-Mott transition in the JCH lattice is now considered in the presence of a synthetic magnetic field $\alpha \neq 0$. This situation differs in several key ways from the nonmagnetic case. Specifically, the field introduces a magnetic lattice which breaks the translational symmetry of the underlying lattice. Hence, with a magnetic field, $\psi_{i}$ cannot be assumed identical across the system. To capture this, a large lattice must be simulated, so that the magnetic and cavity lattices are commensurate. For the magnetic parameter $\alpha=p / q$, there must be $q=N_{x} \times N_{y}$ sites, where $N_{x}\left(N_{y}\right)$ is the number 


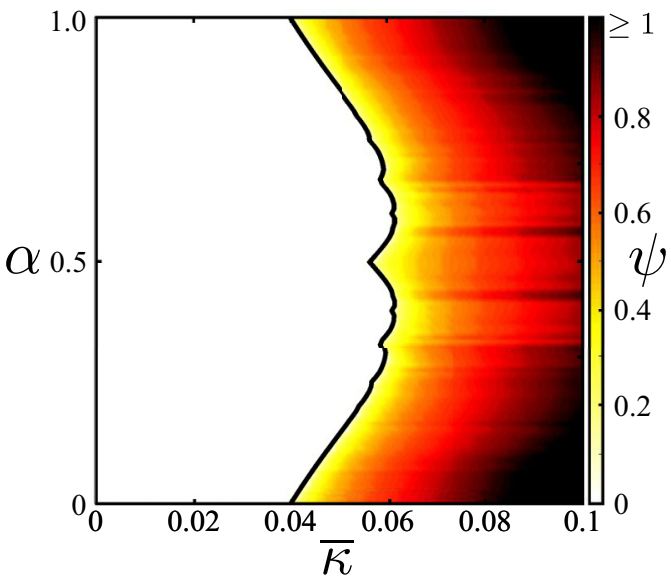

FIG. 4. The maximum superfluid order parameter $(\max [\vec{\psi}])$ as a function of the synthetic magnetic field $\alpha$ and the interactive hopping $\bar{\kappa}$, with $\bar{\mu}=-0.78$ for a $20 \times 20$ lattice. The solid black curve corresponds to the evaluation of the transition between the Mott insulator state and the superfluid state as determined by Eq. (14). We have introduced the following dimensionless parametrization: $\bar{\kappa}=\kappa / \beta, \bar{\mu}=(\mu-\omega) / \beta$, and $\bar{\Delta}=(\omega-\epsilon) / \beta=0$.

of cavities along the $x(y)$ axis. Despite this introducing a synthetic magnetic field does not qualitatively change the phase diagram for the Mott-insulator superfluid phase transition, i.e., at low $\bar{\kappa}$ the lobe structure persists and when $\bar{\kappa}$ is sufficiently large the system undergoes a transition to superfluid state. However, the introduction of a synthetic magnetic field does quantitatively change the phase diagram; see Figs. 3(b) and 3(c). Here we compare the superfluid density at nonzero $\alpha$ to the $\alpha=0$ case (at some $\mu$ and $\kappa$ ) via

$$
\Delta \psi(\alpha)=\frac{\max [\vec{\psi}(\alpha=0)]-\max [\vec{\psi}(\alpha)]}{\max [\vec{\psi}(\alpha=0)]+\max [\vec{\psi}(\alpha)]+\epsilon},
$$

where we have introduced $\epsilon$ to avoid divide by zero errors, but which plays no physical role. Specifically we see that the introduction of a synthetic magnetic field changes (for a fixed chemical potential) the strength of intercavity hopping required to transition from the Mott phase to the superfluid phase.

To quantify how the synthetic magnetic field changes the boundary between the superfluid and Mott phase in Fig. 3 we have plotted, in Fig. 4, $\max [\vec{\psi}]$ as a function of $\bar{\kappa}$ and $\alpha$, with $\bar{\mu}=-0.78$. This shows that increasing the synthetic magnetic field from zero (half a) flux quanta per site to half a (one) flux quanta per site the superfluid Mott insulator boundary is pushed to larger (lower) values of the intercavity hopping.

In the vicinity of the Mott insulator superfluid phase boundary the superfluid parameters $\psi_{i}$ are very small across the entire system. Perturbatively expanding about $\psi_{i}$ it is possible to determine the boundary between the superfluid and Mott phases. Specifically, consider the single site effective Hamiltonian for site $i$ :

$$
H_{\mathrm{eff}, i}^{\mathrm{JCH}}=H_{i}^{\mathrm{JC}}-\mu L_{i}-\kappa \sum_{j}\left[\left(a_{i}^{\dagger}-\frac{1}{2} \psi_{i}^{\star}\right) \Psi_{i}+\text { H.c. }\right]
$$

where $\Psi_{i}=\sum_{j} \kappa_{i j} \psi_{j} / \kappa$ and we have assumed that the magnitude of the hopping rate is the same between sites, i.e., $\left|\kappa_{i j}\right|=\kappa$. Treating the $\psi_{i}$ as perturbative parameters we find

$$
E_{i}(\vec{\psi})=E_{i}^{(0)}-r_{n} \kappa^{2}\left|\Psi_{i}\right|^{2}-\frac{\kappa}{2}\left(\psi_{i}^{\star} \Psi_{i}+\text { H.c. }\right)
$$

where

$$
r_{n}=\sum_{\gamma= \pm} \frac{|\langle\gamma, n+1|a|-, n\rangle|^{2}}{E_{n+1, \gamma}-E_{n,-}-\mu}+\frac{|\langle\gamma, n-1|a|-, n\rangle|^{2}}{E_{n-1, \gamma}-E_{n,-}-\mu}
$$

arises from the second order perturbative corrections to the energy. The full energy can then be written as

$$
E(\vec{\psi})=\sum_{i} E_{i}^{(0)}+\kappa \vec{\psi}^{\dagger} \kappa(\alpha) \vec{\psi}-r_{n} \kappa^{2} \vec{\psi}^{\dagger} \kappa^{2}(\alpha) \vec{\psi}
$$

where $\vec{\psi}$ is a vector of the $\psi_{i}$ and the elements of the matrix $\boldsymbol{\kappa}$ parameterize the phase accumulated, due to the synthetic magnetic field, in hopping between sites. The location of the Mott insulator superfluid transition occurs when some nonzero values for $\psi_{i}$ gives an energy lower than the energy of the Mott lobe, i.e.,

$$
\kappa \vec{\psi}^{\dagger} \boldsymbol{\kappa} \vec{\psi}-r_{n} \kappa^{2} \vec{\psi}^{\dagger} \kappa^{2} \vec{\psi}<0
$$

The solid line in Fig. 4 shows how the phase boundary between the Mott insulator and superfluid changes as a function of the hopping and $\alpha$, as determined from the above equation. As expected, we find very good agreement between this perturbative calculation and the full mean-field calculation in determining the boundary between the two regimes.

The solutions for Eq. (14) occur for $\kappa>1 / \kappa f(\alpha)$, where $f(\alpha)$ is the maximum eigenvalue of $\kappa(\alpha)$. As noted in studies of the Bose-Hubbard model [23], $f(\alpha)$ is the outline of the Hofstadter butterfly. Thus the suppression of the phase transition comes from the frustration induced by the incommensurate lattice or magnetic lengths. This leads in general to fractal structures (as seen in the Hofstadter butterfly) and is similarly reflected in the phase boundary seen here.

In Fig. 4 we note that the some of the very fine structure in $f(\alpha)$ is visible, both in the location of the boundary and within the superfluid region. This structure is perhaps over represented in the figure since, in finding the ground-state wave function, we limited the system size to a $20 \times 20$ lattice. The presence of a butterfly-like structure has already been observed in experiments [24,25], including some involving photons [26]. As expected [27], small fluctuations in the system lead to a blurring of the fractal structure, and so the very fine structure seen in Fig. 4 would be challenging to resolve in any experiment.

Our analysis of the JCH system is undertaken in the language of a particle-conserving condensed matter system. How does the existence or absence of long-range correlations in the ground state of this model translate to a realistic photonic system: one driven by a laser (or some external voltage in the case of circuit QED) with dissipation and decoherence? Essentially, spacial correlations within the system will be exponentially vanishing past a critical point in the atomic interaction (Mott phase). The ground state of our model system is simply one of many channels open to photonic states within the optical system. So for some configuration, one should 
consider the chemical potential as a means by which to look at the spectrum of states that will be occupied.

From this perspective our results regarding the response of a Jaynes-Cummings-Hubbard lattice is readily interpretable: Frustration induced by the magnetic field amplifies the effect of interactions acting to suppress long range correlations in the photonic fluid. This is a readily observable phenomena in isolation. However, since various open-system effects will have similar effects, disentangling these may prove difficult. The dynamics of the open JCH system are far richer [16] and more complicated than the situation we have considered. The effects that we identify in this work, in regards to the response of the superfluid-Mott phase transition to magnetic fields, translates readily into the open systems context. As with the nonmagnetic case [17], one should expect to find phenomena dependent on the various open system effects (phenomena not identified here). However, we leave investigation into this for future investigations.

\section{VORTICES}

In dilute Bose gas superfluids the introduction of synthetic magnetic fields [28-32], or the application of rotation to break time reversal symmetry, has led to the observation of single vortices [33], vortex lattices [34-36], and the prediction of the emergence of fractional quantum Hall states [28,37-42]. The application of a synthetic magnetic field in a JCH lattice is expected to produce similar effects in the superfluid state. Considerable effort has already been applied to the study of of fractional quantum Hall states [8] in the "high" synthetic magnetic field regime, where the number of flux quanta though the lattice is larger than the number of excitations. However, less attention has been paid to the emergence of a vortex and vortex lattice states in the "low" synthetic magnetic field regime. Below we demonstrate that the JCH system does admit vortex and vortex lattice solutions in the superfluid regime, upon the introduction of a synthetic magnetic field.

In the context of the mean-field description of the JCH model, the local order parameter shares all the characteristics of a superfluid. Figures 5(a) and 5(b) show the magnitude of the mean-field order parameter (a) and its phase (b) for a periodic lattice with a single flux quantum penetrating $64 \times 64$ sites. Minimizing the mean-field energy functional [Eq. (11)] results in a single vortex structure where the superfluid density rises monotonically from the center of the vortex core and the superfluid phase rotates by $2 \pi$.

Figure 6 shows a cross section of a single vortex for increasing values of the chemical potential. As the chemical potential increases at a set $\kappa$ and $\Delta$, the superfluid density increases. This leads to a stronger repulsive force within the superfluid, and, as in the case of conventional superfluids, a decreasing core size for the vortex. Note that, since the Jaynes-Cummings interaction increases slowly with excitation (square root), the effect of higher $|\psi|$ is diminishing as $\mu$ rises.

At higher synthetic magnetic fields one expects multiple vortices to be admitted into the superfluid. In the absence of an underlying lattice structure the vortices will arrange themselves into a triangular Abrikosov vortex lattice. This configuration arises, in the presence of a local repulsive nonlinearity, to minimize the self-interaction of the superfluid
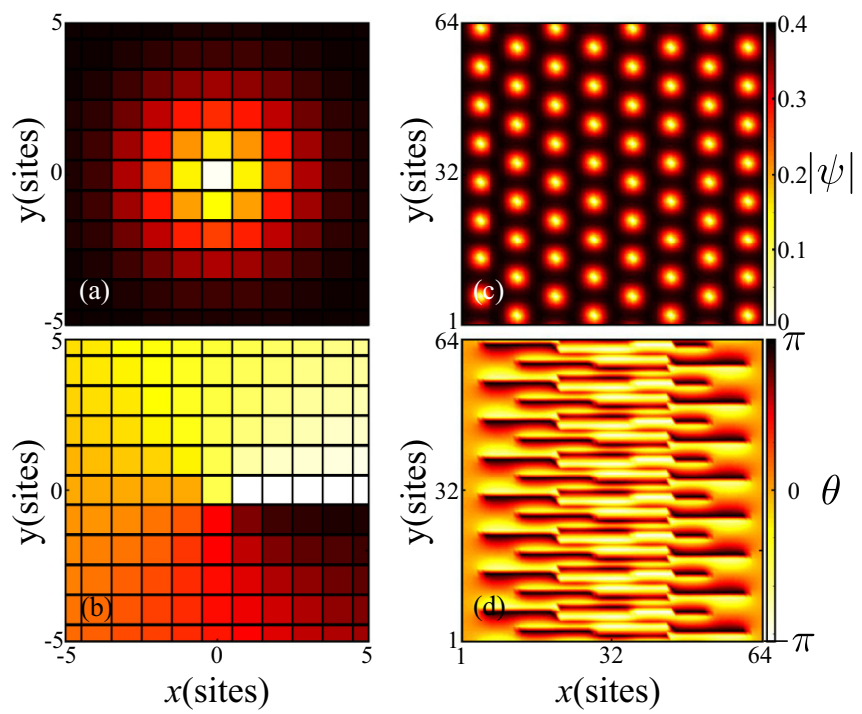

FIG. 5. Superfluid density $|\psi|$ (a, c) and phase (b, d) on a $64 \times 64$ lattice, with $\bar{\kappa}=0.045, \bar{\Delta}=0$ and $\bar{\mu}=-0.78$ for $\alpha=1 / 64^{2}(\mathrm{a}, \mathrm{b})$ and $\alpha=1 / 64$ (c, d).

at a given density of vortices. Figures 5(c) and 5(d) show that energy minimization of the mean-field $\mathrm{JCH}$, in the presence of a synthetic magnetic field, also leads to the formation of an Abrikosov vortex lattice state.

\section{CONCLUSIONS}

We have shown that the introduction of a synthetic magnetic field to the JCH model modifies the boundary between the Mott insulator and superfluid regimes. This modification arises from a competition between the magnetic lattice and the spatial lattice. Additionally, we predict that in the superfluid regime the introduction of a synthetic magnetic field leads to the formation of vortices which, due to the local nonlinear atom-photon interaction, forms a triangular lattice in the ground state.

This work opens up possible avenues for future research into the role of the $\mathrm{JCH}$ atom-cavity interaction in frustrating the formation of triangular Abrikosov vortex lattices and, since this is an inherently two-dimensional system, the possibility of observing a BKT transition.

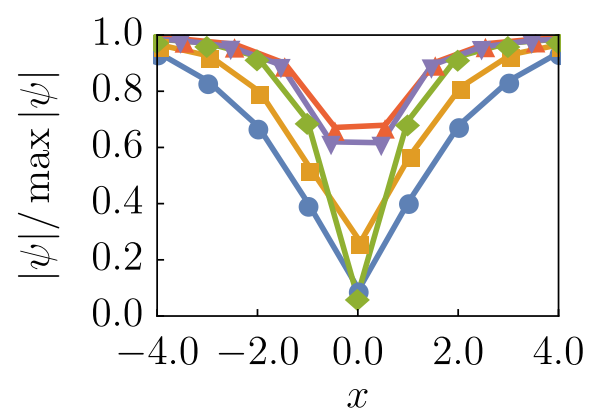

FIG. 6. Superfluid density $|\psi|$ on a $64 \times 64$ lattice, with $\bar{\kappa}=$ $0.045, \bar{\Delta}=0$ and $\bar{\mu}$ blue: $-0.78(\bullet)$, orange: $-0.7(\boldsymbol{\square})$, green: -0.6 $(\diamond)$, red: $-0.5(\mathbf{\Delta})$, purple: $-0.4(\mathbf{\nabla})$. 
[1] M. J. Hartmann, F. G. S. L. Brandão, and M. B. Plenio, Nat. Phys. 2, 849 (2006).

[2] A. D. Greentree, C. Tahan, J. H. Cole, and L. C. L. Hollenberg, Nat. Phys. 2, 856 (2006).

[3] D. G. Angelakis, M. F. Santos, and S. Bose, Phys. Rev. A 76, 031805(R) (2007).

[4] B. Bujnowski, J. K. Corso, A. L. C. Hayward, J. H. Cole, and A. M. Martin, Phys. Rev. A 90, 043801 (2014).

[5] J. Quach, M. I. Makin, C.-H. Su, A. D. Greentree, and L. C. L. Hollenberg, Phys. Rev. A 80, 063838 (2009).

[6] D. Gerace, H. E. Türeci, A. Imamoglu, V. Giovannetti, and R. Fazio, Nat. Phys. 5, 281 (2009).

[7] J. Q. Quach, C.-H. Su, A. M. Martin, A. D. Greentree, and L. C. L. Hollenberg, Opt. Express 19, 11018 (2011).

[8] E. Kapit, M. Hafezi, and S. H. Simon, Phys. Rev. X 4, 031039 (2014).

[9] D. Rossini and R. Fazio, Phys. Rev. Lett. 99, 186401 (2007).

[10] J. Koch and K. Le Hur, Phys. Rev. A 80, 023811 (2009).

[11] M. Hohenadler, M. Aichhorn, S. Schmidt, and L. Pollet, Phys. Rev. A 84, 041608(R) (2011).

[12] D. Rossini, R. Fazio, and G. Santoro, Europhys. Lett. 83, 47011 (2008).

[13] J. Jin, R. Fazio, and D. Rossini, EPJ Quantum Tech. 2, 5 (2015).

[14] H. Zheng and Y. Takada, Phys. Rev. A 84, 043819 (2011).

[15] A. Hu, T. E. Lee, and C. W. Clark, Phys. Rev. A 88, 053627 (2013).

[16] I. Carusotto and C. Ciuti, Rev. Mod. Phys. 85, 299 (2013).

[17] A. Le Boité, G. Orso, and C. Ciuti, Phys. Rev. A 90, 063821 (2014).

[18] F. Haldane and S. Raghu, Phys. Rev. Lett. 100, 013904 (2008).

[19] J. Cho, D. G. Angelakis, and S. Bose, Phys. Rev. Lett. 101, 246809 (2008).

[20] J. Koch, A. A. Houck, K. L. Hur, and S. M. Girvin, Phys. Rev. A 82, 043811 (2010).

[21] A. R. Kolovsky, Europhys. Lett. 93, 20003 (2011).

[22] R. O. Umucalilar and I. Carusotto, Phys. Rev. A 84, 043804 (2011).
[23] M. Ö. Oktel, M. Niţă, and B. Tanatar, Phys. Rev. B 75, 045133 (2007).

[24] C. R. Dean, L. Wang, P. Maher, C. Forsythe, F. Ghahari, Y. Gao, J. Katoch, M. Ishigami, P. Moon, M. Koshino et al., Nature (London) 497, 598 (2013).

[25] T. Schlösser, K. Ensslin, J. P. Kotthaus, and M. Holland, Europhys. Lett. 33, 683 (1996).

[26] U. Kuhl and H.-J. Stöckmann, Phys. Rev. Lett. 80, 3232 (1998).

[27] D. Hofstadter, Phys. Rev. B 14, 2239 (1976).

[28] J. Ruostekoski, G. V. Dunne, and J. Javanainen, Phys. Rev. Lett. 88, 180401 (2002).

[29] D. Jaksch and P. Zoller, New J. Phys. 5, 56 (2003).

[30] E. J. Mueller, Phys. Rev. A 70, 041603 (2004).

[31] I. B. Spielman, Phys. Rev. A 79, 063613 (2009).

[32] D. R. Murray, P. Öhberg, D. Gomila, and S. M. Barnett, Phys. Rev. A 79, 063618 (2009).

[33] Y.-J. Lin, R. L. Compton, K. Jiménez-García, J. V. Porto, and I. B. Spielman, Nature (London) 462, 628 (2009).

[34] K. W. Madison, F. Chevy, W. Wohlleben, and J. Dalibard, Phys. Rev. Lett. 84, 806 (2000).

[35] K. W. Madison, F. Chevy, V. Bretin, and J. Dalibard, Phys. Rev. Lett. 86, 4443 (2001).

[36] E. Hodby, G. Hechenblaikner, S. A. Hopkins, O. M. Maragò, and C. J. Foot, Phys. Rev. Lett. 88, 010405 (2001).

[37] N. R. Cooper and N. K. Wilkin, Phys. Rev. B 60, R16279(R) (1999).

[38] N. K. Wilkin and J. M. F. Gunn, Phys. Rev. Lett. 84, 6 (2000).

[39] N. R. Cooper, N. K. Wilkin, and J. M. F. Gunn, Phys. Rev. Lett. 87, 120405 (2001).

[40] V. Bretin, S. Stock, Y. Seurin, and J. Dalibard, Phys. Rev. Lett. 92, 050403 (2004).

[41] V. Schweikhard, I. Coddington, P. Engels, V. P. Mogendorff, and E. A. Cornell, Phys. Rev. Lett. 92, 040404 (2004).

[42] M. A. Baranov, K. Osterloh, and M. Lewenstein, Phys. Rev. Lett. 94, 070404 (2005). 


\section{University Library}

\section{- M M N E R VA A gateway to Melbourne's research publications}

Minerva Access is the Institutional Repository of The University of Melbourne

Author/s:

Hayward, ALC;Martin, AM

Title:

Superfluid-Mott transitions and vortices in the Jaynes-Cummings-Hubbard lattices with timereversal-symmetry breaking

Date:

2016-02-22

Citation:

Hayward, A. L. C. \& Martin, A. M. (2016). Superfluid-Mott transitions and vortices in the Jaynes-Cummings-Hubbard lattices with time-reversal-symmetry breaking. PHYSICAL REVIEW A, 93 (2), https://doi.org/10.1103/PhysRevA.93.023828.

Persistent Link:

http://hdl.handle.net/11343/116642 